\title{
Fluorescence spectra of laser-excited YO molecules in a $\mathrm{H}_{2}-\mathrm{O}_{2}-\mathbf{A r}$ flame
}

\author{
T. Wijchers, H. A. Dijkerman, P. J. Th. Zeegers and C. Th. J. Alkemade \\ Fysisch Laboratorium, Rijksuniversiteit Utrecht, Princetonplein 5, 3508 TA Utrecht, The Netherlands
}

(Received 23 November 1979)

\begin{abstract}
CW dye laser induced fluorescence emission and thermal emission spectra of YO-molecules in a $1 \mathrm{~atm} \mathrm{H}_{2}-\mathrm{O}_{2}-\mathrm{Ar}$ flame of $2430 \mathrm{~K}$ were recorded simultaneously. Narrow band laser excitation was applied to four rotational lines in the $(1,1)$ Q-branch of the $A^{2} \Pi_{3 / 2} \rightarrow X^{2} \Sigma^{+}$transition and broadband excitation was applied to several separate $Q$-branches of the $A^{2} \Pi_{1 / 2,3 / 2} \rightarrow X^{2} \Sigma^{+}$transitions. From the differences between the fluorescence emission spectra and thermal emission spectra, we conclude that collisional de-excitation of an excited vibronic level takes place by vibrational relaxation, decay to the electronic ground state and by intermultiplet transfer in order of increasing probability.
\end{abstract}

\section{INTRODUCTION}

As a CONIINUATION of the work already done in this laboratory on determining collisional rate constants and mixing cross sections $[1,2]$ we are currently trying,to determine relative rate constants of collisional transitions between vibronic levels of diatomic metal compounds in flames at $1 \mathrm{~atm}$ pressure. These relative rate constants can be derived from the ratios between $n_{i}(=$ the thermal population of vibronic level $i$ ) and $\delta n_{i}$ (the population increase of the same level due to laser excitation).

The aim of the present investigation is to show that a comparison of fluorescence and thermal emission spectra makes the determination of these ratio's feasible.

In their exploratory investigation BLACKBURN et al. [3] obtained fluorescence emission spectra of $\mathrm{SrOH}, \mathrm{CaOH}, \mathrm{CrO}, \mathrm{MnO}$ and $\mathrm{C}_{2}$ in a flame using a $\mathrm{CW}$ dye laser as excitation source. Triatomic molecular spectra are usually very complex due to overlapping bands and are thus less suitable for determining collisional rate constants. We decided to investigate a diatomic molecule which has sufficiently strong absorption bands in a wavelength range which can be covered by the tuning range of a dye laser. The thermal emission spectrum of $\mathrm{YO}$ shows strong $\Delta v=0$ vibronic bands between 5970 and $6280 \AA$ of the $A^{2} \mathrm{II} \rightarrow X^{2} \Sigma^{+}$transitions. In both sequences, the Q-branches are relatively narrow and clearly superimposed on the P- and R-branches. A rhodamine $6 \mathrm{G}$ operated dye laser is suitable for covering this wavelength range. The dissociation energy of $7.31 \mathrm{eV}$ [4] will assure an efficient production of $\mathrm{YO}$ from yttrium salts in a $\mathrm{H}_{2}-\mathrm{O}_{2}-\mathrm{Ar}$ flame provided no other yttrium compounds with still higher dissociation energy can be produced in this flame. UHLER and ÅKERLIND [5] have rotationally analyzed the branches of the vibronic $(0,0)$ transition between the $A^{2} \Pi_{1 / 2,3 / 2}$ and $X^{2} \Sigma^{+}$levels of $Y O$ molecules in an air-acetylene flame. The distances which they found between the rotational lines are smaller than the resolving power of our monochromator but many of them are larger than the linewidths of the CW dye laser used. Recently BERNARD et al. published a more extended rotation analysis of the $A^{2} \Pi \rightarrow X^{2} \Sigma^{+}$system of YO with higher resolving power [6]. They used the Fourier

[1] C. A. Van Disk, Ph. D. Thesis, University of Utrecht (1978).

[2] P. L. Lijnse, P. J. Th. Zeegers and C. TH. J. Alkemade, J. Quant. Spectrosc. Radiat. Transfer 13, 1033 (1973).

[3] M. B. Blackdurn, J. M. Mermet and J. D. Winefordner, Spectrochim. Acta 34B, 847 (1978).

[4] R. J. Ackermann, E. G. Rank and R. J. Thom, J. Chem. Phys. 40, 883 (1964).

[5] U. Uhler and L. ÅkerLind, Ark. Fys. 19, 1 (1961).

[6] A. Bernard, R. Bacis and P. Luc, Astroph. J. 227, 338 (1979). 
transform spectroscopy method to obtain spectra of YO molecules in a cooled hollow cathode discharge tube.

The population of a vibrational level is achieved most efficiently by exciting the Q-branch of a specific vibronic transition. This excitation can be realised by exciting either one rovibronic line or a number of rovibronic lines in this Q-branch. Using the single-line excitation method one can also compare the rotational redistribution rate with the optical de-excitation rate.

In order to be sure that only one rotational line is excited by the laser we have to know the widths of and distances between the rotational lines of the vibronic transitions involved in this study. The set-up for single-line excitation with a narrow band laser which we use to record a fluorescence excitation spectrum for measuring these widths and distances is described in Section 2.4.2. In Sections 2.4.3 and 2.4.4 set-ups are described for population of a vibronic level by single-line excitation and multi-line excitation, respectively.

We shall give some examples of fluorescence emission spectra which resulted from single- and multi-line excitation and present conclusions which could be drawn from the comparison of the fluorescence emission spectra and the thermal emission spectra.

\section{EXPERIMENTAL}

\subsection{General set-up}

The scheme of the whole set-up is given in Fig. 1. A pre-mixed $1 \mathrm{~atm} \mathrm{H}_{2}-\mathrm{O}_{2}-\mathrm{Ar}$ flame Fl containing $\mathrm{YO}$ molecules was traversed 10 times by a collimated $\mathrm{CW}$ dye laser beam. Two prisms were used for retroreflection in the horizontal plane; the $90^{\circ}$ edge of one prism was truncated to $1.5 \mathrm{~mm}$ and then a small entrance prism was attached. To an adjoining reflecting side a second small prism was cemented for deflection of the multiply-reflected beam.

The irradiated volume of $\mathrm{Fl}$ was imaged onto the entrance slit of a $1 \mathrm{~m}$ monochromator $\mathrm{M}$ (McPherson 2051) by means of an imaging system $\mathrm{L}$. The intensities of fluorescence and thermal emission radiation were modulated at $320 \mathrm{~Hz}$ with chopper Ch 1 (PAR 222) and detected via M by a cooled photomultiplier D1 (EMI 9558 QA). The beam intensity from dye laser DL (Coherent 590) was modulated either by chopper Ch 2 at $125 \mathrm{~Hz}$ or otherwise (Section 2.4.1), and collimated by lens CL.

The wavelength of DL could be changed by applying a signal from generator $G$ through driving amplifier DA to DL (Section 2.4.1).

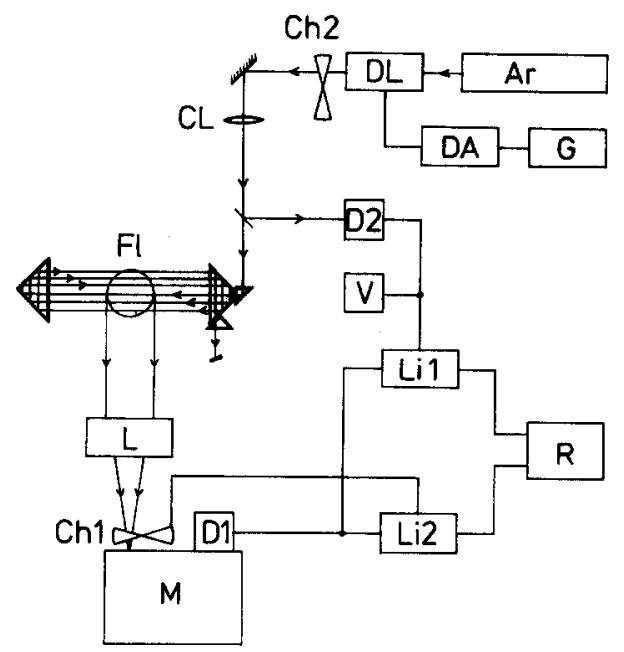

Fig. 1. Block scheme of the complete set-up for excitation and thermal emission measurements. Ar: Argon-ion laser, DL: dye laser, DA: driving amplifier, G: Generator, $\mathrm{Ch} 1$ and $\mathrm{Ch} 2$ : choppers, CL: collimation lens, F1: flame, $\mathrm{L}$ : imaging system, $\mathrm{M}$ : monochromator, D1 and D2: detectors, Li1 and Li2: lock-ins, R: two-channel stripchart recorder, V: A.C. voltmeter. 
Detector D2 was used as a monitor for the laser power and provided lock-in Li1 (lab. made) with a reference signal for phase-sensitive detection of fluorescence. Lock-in Li2 (lab. made), which was connected with D1, obtained its reference signal from chopper $\mathrm{Ch} 1$ and measured the sum of the fluorescence signals and thermal emission signals at $320 \mathrm{~Hz}$.

Since fluorescence and thermal emission were measured simultaneously, we did not need to monitor the YO density.

The output signals of both lock-in amplifiers were fed into a 2 channel stripchart recorder (Kipp BD 9).

\subsection{Flame set-up}

A pre-mixed $\mathrm{H}_{2}-\mathrm{O}_{2}-$ Ar flame at 1 atm was produced with a laboratory-made Méker burner similar to the type described in [7]. We measured the temperature of the flame, using the visual $\mathrm{Na}$-line reversal method and found it to be $2420 \pm 15 \mathrm{~K}$.

The burner head had 148 holes of $0.7 \mathrm{~mm}$ diameter for the central flame and 440 holes with the same diameter for the mantle flame. At the centre of detection, $45 \mathrm{~mm}$ above the burner head, the diameters of the central and mantle flame were about 20 and $40 \mathrm{~mm}$, respectively.

The gases were supplied via calibrated rotameter-type flowmeters, the flows being regulated by needle valves.

A commercial pneumatic nebulizing system (Varian Techtron) with fixed nebulizer, attached to the bottom of the vertical gas-mixing tube of the central flame, provided the central flame with an $\mathrm{Y}\left(\mathrm{ClO}_{4}\right)_{3}$ solution in ethanol. The $\mathrm{Y}$ concentration was about $0.6 \mathrm{~g} \mathrm{l}^{-1}$. Argon (6.81. min $\left.{ }^{-1}\right)$ and hydrogen $\left(3.81 . \mathrm{min}^{-1}\right)$ together served as driving gas for nebulizing the solution; oxygen was added separately in the spray chamber of the nebulizing system. The oxygen flow was adjusted at $2.81 . \mathrm{min}^{-1}$ to obtain a maximum YO signal.

\subsection{Detection set-up for fluorescence and thermal emission}

For relative measurements the dimensions of the imaging system are not critical and the system can be kept simple. The system described below has been made for future quantitative measurements but was used here because of its high optical conductance.

In Figs. 2( $a$ and $b$ ) the imaging system $L$ is shown in the horizontal and vertical plane, respectively.

In the horizontal plane the focal point of the cylindrical lens system, consisting of L2 (focal length $=150 \mathrm{~mm}$ ) and $\mathrm{L} 3$ (focal length $=-330 \mathrm{~mm}$ ), coincides with the entrance slit of the monochromator. Consequently the whole cross section of the central flame was imaged on the slit at an angle of $S / f \mathrm{rad}, S$ and $f$ being the slitwidth and focal length of system L2, L3, respectively. A back mirror Mi was used to enhance the optical signal.

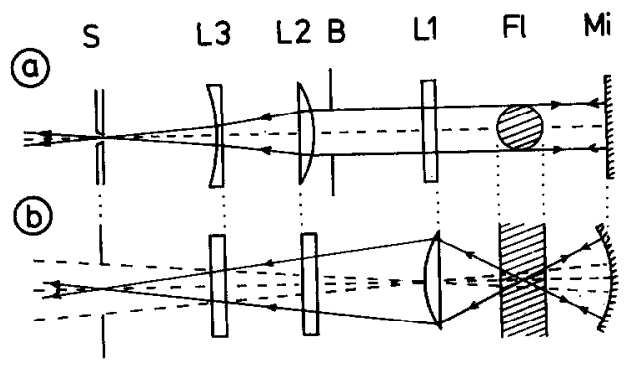

Fig. 2. Imaging system between flame FL and monochromator slit $\mathrm{S}$, (a) in the horizontal plane, (b) in the vertical plane. L1, L2 and L3: cylindrical lenses, Mi: cylindrical mirror, B: baffle.

Dimensions are not to scale.

[7] TJ. Hollander, Ph. D. Thesis, University of Utrecht (1964). 
The focal length of system L2, L3, was adjusted at $225 \mathrm{~mm}$ so that the radiative beam from the central flame which passed the entrance slit would enter within the aperture of the monochromator. Radiation from outside this beam which could have entered the entrance slit was screened off by baffle $B$.

In the vertical plane, radiation from the flame centre was imaged, either directly or indirectly via the cylindrical back mirror $\mathrm{Mi}$, by cylindrical lens $\mathrm{L} 1$ in $\mathrm{S}$.

The chopper wheel of Ch1 was placed at a short distance from S. Spectral selection was obtained with a $1 \mathrm{~m}$ Cerny-Turner scanning monochromator provided with a 1200 lines $/ \mathrm{mm}, 102 \times 102 \mathrm{~mm}^{2}$ grating blazed at $5000 \AA$. The width and height of entrance and exit slits were 0.2 and $15 \mathrm{~mm}$, respectively. By turning a flat mirror at an angle of $45^{\circ}$ in the beam inside the monochromator the spectra could be observed visually through an eyepiece. This eyepiece, provided with a $10 \mathrm{~mm}$ long scale divided into 200 intervals, was also used to calibrate the laser sweep width (see Section 2.4.4).

\subsection{Laser set-up}

2.4.1 Basic equipment. A CW dye laser was pumped by an Ar-ion laser (Spectra Physics) with $2.5 \mathrm{~W}$ output power at $5145 \AA$. The dye laser was provided with a 2-plate birefringent filter for wavelength selection.

For wavelength scanning an intracavity etalon-tilting device was made as shown in Fig. 3. The axis of tilting a was horizontal, and perpendicular to the laser beam direction. By means of bar $b$ and leafspring $s 2$, axis a was adjustably attached to a vertical thread bar $t$. The latter connected loudspeaker coil $c$ to leafspring $s 1$.

Wavelength scanning was accomplished by applying a variable current through the loudspeaker coil from driving amplifier DA (see Fig. 1). This amplifier was provided with two d.c. coupled inputs. The output of DA could deliver either a voltage or a current proportional to the input voltage.

A constant flow of nitrogen under the dye laser cover prevented polluted air from affecting the laser mirrors under high radiant density conditions. This enabled us to use the laser at full power specifications for many days.

2.4.2 Laser set-up for the fluorescence excitation spectrum. To obtain the fluorescence excitation spectrum a $0.5 \mathrm{~mm}$ uncoated solid etalon was added to the basic lay-out. With this etalon the linewidth was about $70 \mathrm{~m} \AA$. A $5 \mathrm{~mm}$ etalon of the same type could be added, resulting in a linewidth of $20 \mathrm{~m} \AA$.

Driving amplifier DA, switched in the voltage-to-current mode, was fed with a slowly varying triangular voltage signal from generator $G$ (see Fig. 1) which had a repetition rate of about $0.003 / \mathrm{s}$. During a single sweep the birefringent filter was re-adjusted continuously for maximum power. To prevent a change in power due to variation of the etalon tilting angle, we made use of a Kerr-effect power stabilizer placed outside the laser cavity. This stabilizer, made from an ADP crystal (Lasermetrics $3030 \mathrm{XFW}$ ) in our laboratory, kept the laser power at a constant value corresponding to the maximum etalon tilting angle. The laser power was modulated sinusoidally by applying a $490 \mathrm{~Hz}$ external signal to the power stabilizer.

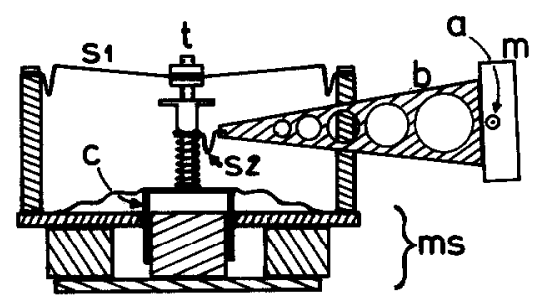

Fig. 3. Etalon-tilting mechanism. Leafspring $\mathrm{s} 1$ and its vertical mounts are turned $90^{\circ}$ for the sake of clarity. The laser beam travels in the plane of the picture. Dimensions are not to scale. c: loudspeaker coil, $t$ : thread bar, s2: leafspring, b: bar, a: axis, m: etalon mount, ms: loudspeaker magnetic system. 
For practical reasons, the detection set-up described in Section 2.3 was used but chopper Ch1 was omitted. The spectral sensitivity of the fluorescence radiation detection was determined by the spectral sensitivity of the photomultiplier and the spectral transmission of the optical components between flame and photomultiplier. Hence the detection region reached from 3000 to $7000 \AA$ and was spectrally integrated by the monochromator. The latter was set at zero order and the slits were wide open at $2 \times 15 \mathrm{~mm}^{2}$ for maximum optical conductance.

Lock-in amplifier Li1 detected the spectrally integrated fluorescence signal at $490 \mathrm{~Hz}$.

The slowly varying triangular voltage was recorded simultaneously with the excitation spectrum through the second channel of the recorder. Since this voltage is a measure for the tilting angle, the latter was defined together with the fluorescence excitation spectrum.

After the excitation spectrum and triangular voltage had been recorded, a repeated laser sweep was carried out. The second-channel pen recording the triangular voltage, followed again its same track on the stripchart where already the excitation spectrum was recorded but now the first-channel pen recorded the non-stabilized laser power while the birefringent filter was kept stationary.

This recording through the first channel showed a series of minima separated by the known free-spectral-range of the etalon. In this way a wavelength scale was obtained being $2.34 \AA$ at mean tilting angle.

2.4.3 Laser set-up for single-line excitation. For single-line excitation we used the basic equipment described in Section 2.4 .1 to which the $0.5 \mathrm{~mm}$ solid uncoated etalon had been attached.

Since only small variations in wavelength were needed, leafspring $s 1$, mentioned in Section 2.4 .2 , was replaced by a stiffer one by means of which the wavelength-sweep equipment was operated with not too small a generator voltage.

The laser wavelength was stabilized on a given rotational line with wavelength $\lambda_{0}$ by the widely used $\lambda$-stabilization technique. With this technique the laser wavelength was slightly modulated around $\lambda_{0}$, here about $20 \mathrm{~m} \AA$ at a frequency of $85 \mathrm{~Hz}$. The resulting fluorescence signal was phase-sensitively detected and the d.c. signal was fed into one input of DA while the other input of DA was used for the wavelength modulation. At correct polarity of the phase sensitive detection the laser central wavelength was kept at $\lambda_{0}$.

2.4.4 Laser set-up for multi-line excitation. Multi-line excitation was accomplished by fast periodic sweeping of the wavelength $\lambda$ of the laser (bandwidth $0.6 \AA$ ) with an amplitude that was large enough to cover the clearly superimposed part of the absorption band of a chosen Q-branch. If there is no saturation, it can be shown that this method of excitation, combined with averaging of the fluorescence intensity, has the same effect as excitation by a broad-band source with uniform spectral intensity. During the sweeping of the laser wavelength, the instantaneous laser power as well as $\mathrm{d} \lambda / \mathrm{d} t$ are generally dependent on $\lambda$.

The relative power variations were reduced to about $1.5 \%$ when a $0.1 \mathrm{~mm}$ uncoated solid etalon was used and the thickest plate of the birefringent filter was removed

To make $\mathrm{d} \lambda / \mathrm{d} t$ independent of the $\lambda$ variation, the stiff leafspring $\mathrm{s} 1$ was made non linear by giving it the shape sketched in Fig. 3. As a result the recorded profile produced by the periodically sweeping laser line was rectangular within a statistical error of $3 \%$. The width of this $\lambda$-profile was measured visually as described in Section 2.3 and could be adjusted up to about $10 \AA$.

\section{RESULTS}

\subsection{The fluorescence excitation spectrum}

Despite the limited solid angle of detection an excitation spectrum for the $\left(v^{\prime}, v^{\prime \prime}=v^{\prime}\right)$ $A^{2} \Pi_{1 / 2,3 / 2} \rightarrow X^{2} \Sigma^{+}$transitions was obtained from $v^{\prime}=0$ to $v^{\prime}=7$ with the set-ups 
described in Sections 2.3 and 2.4.2. Rotational lines are well separated everywhere in the spectrum except in parts of the $v^{\prime}=0$ Q-branches in both doublet components.

In [5] the rotational lines of these Q-branches were partly resolved and identified. We obtained the same resolution in these Q-branches. A part of the fluorescence excitation spectrum showing the branches $(0,0)$ and $(1,1)$ of the $A^{2} \Pi_{3 / 2} \rightarrow X^{2} \Sigma^{+}$ transition is presented in Fig. 4. Note that the wavelength scale is not linear. The width of the rotational lines convoluted with the laser band profile is $200 \mathrm{~m} \AA$ or larger.

No further details appeared when the laser band width was reduced to $20 \mathrm{~m} \AA$. Apparently the rotational lines are broadened by Doppler and collisional broadening. This conclusion is in agreement with [6] where the rotational linewidth convoluted with the spectrometer profile was approximately $50 \mathrm{~m} \AA$ under lower pressure (2 Torr).

\subsection{Single-line excited fluorescence emission spectra}

In Figs. 5(a-d) fluorescence spectra of the $A^{2} \Pi_{1 / 2,3 / 2} \rightarrow X^{2} \Sigma^{+}$transitions are shown. Excitation took place separately in the first four lines from the head of the $(1,1)$ $A^{2} \Pi_{3 / 2} \rightarrow X^{2} \Sigma^{+}$Q-branch, respectively. The thermal emission spectrum in the same $\lambda$ region between 5950 and $6250 \AA$ is presented in Fig. 5(e). The vibrational temperature derived from the thermal $Q$-branch emission spectrum of the $A^{2} \Pi_{3 / 2} \rightarrow X^{2} \Sigma^{+}$transition is $2430 \pm 100 \mathrm{~K}$, which is in agreement with the gas temperature of $2420 \pm 15 \mathrm{~K}$ measured with the visual line-reversal method. However, the measured vibrational temperature of the $A^{2} \Pi_{1 / 2} \rightarrow X^{2} \Sigma^{+}$transition is $1970 \pm 20 \mathrm{~K}$. The deviation from the other temperatures may be caused by the fact that the Q-branches measured in this doublet component are less clearly superimposed than in the other doublet component.

There are four significant differences between the fluorescence emission spectra and the thermal emission spectrum.

1. The spectrally integrated fluorescence intensities of the Q-branches decrease more strongly with increasing $v^{\prime}$ for $v^{\prime}>2$.

2. The spectrally integrated intensity of the $A^{2} \Pi_{1 / 2} \rightarrow X^{2} \Sigma^{+}$transition with respect to the spcctrally integrated intensity of the $A^{2} \Pi_{3 / 2} \rightarrow X^{2} \Sigma^{+}$transition is only a little smaller in the fluorescence emission spectrum than in the thermal emission spectrum.

3. Structures marked with arrows in Figs. $5(\mathrm{a}-\mathrm{d})$ in the Q-, P- and R-branches of both doublet transitions occur mainly in the fluorescence spectra of $A^{2} \Pi_{3 / 2} \rightarrow X^{2} \Sigma^{+}$but

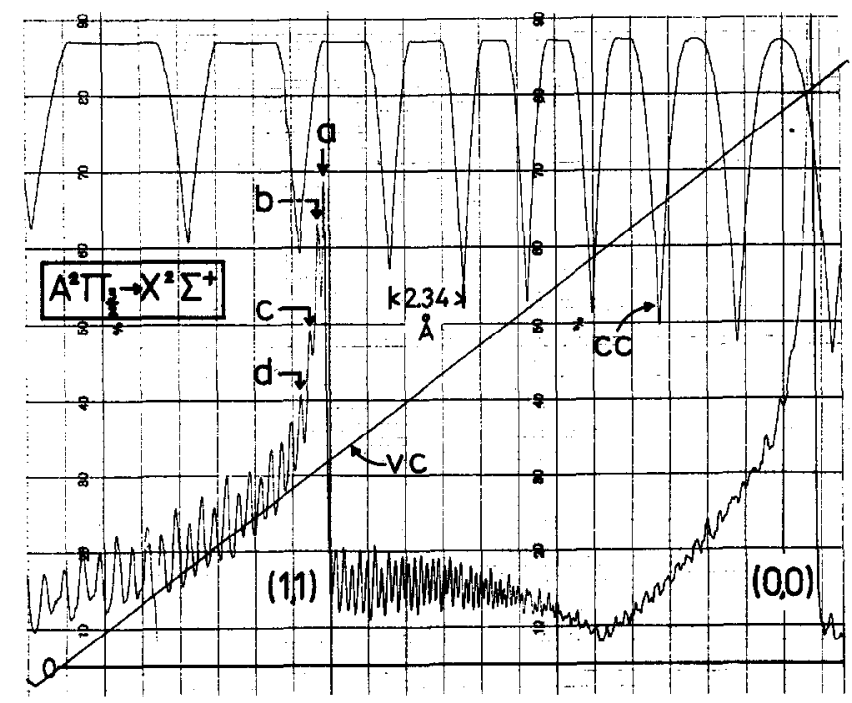

Fig. 4. Fluorescence excitation spectrum of the $Q$-branches $(0,0)$ and $(1,1)$ of the $A^{2} \Pi_{3 / 2} \rightarrow$ $X^{2} \Sigma^{+}$transition. Rotational lines marked with $a, b, c$ and $d$ were excited to produce the fluorescence emission spectra in Figs. 5(a, b, c and d) respectively (see Section 3.2). For an explanation of the wavelength calibration curve $\mathrm{cc}$ and the generator voltage curve vc see Section 2.4.2. 


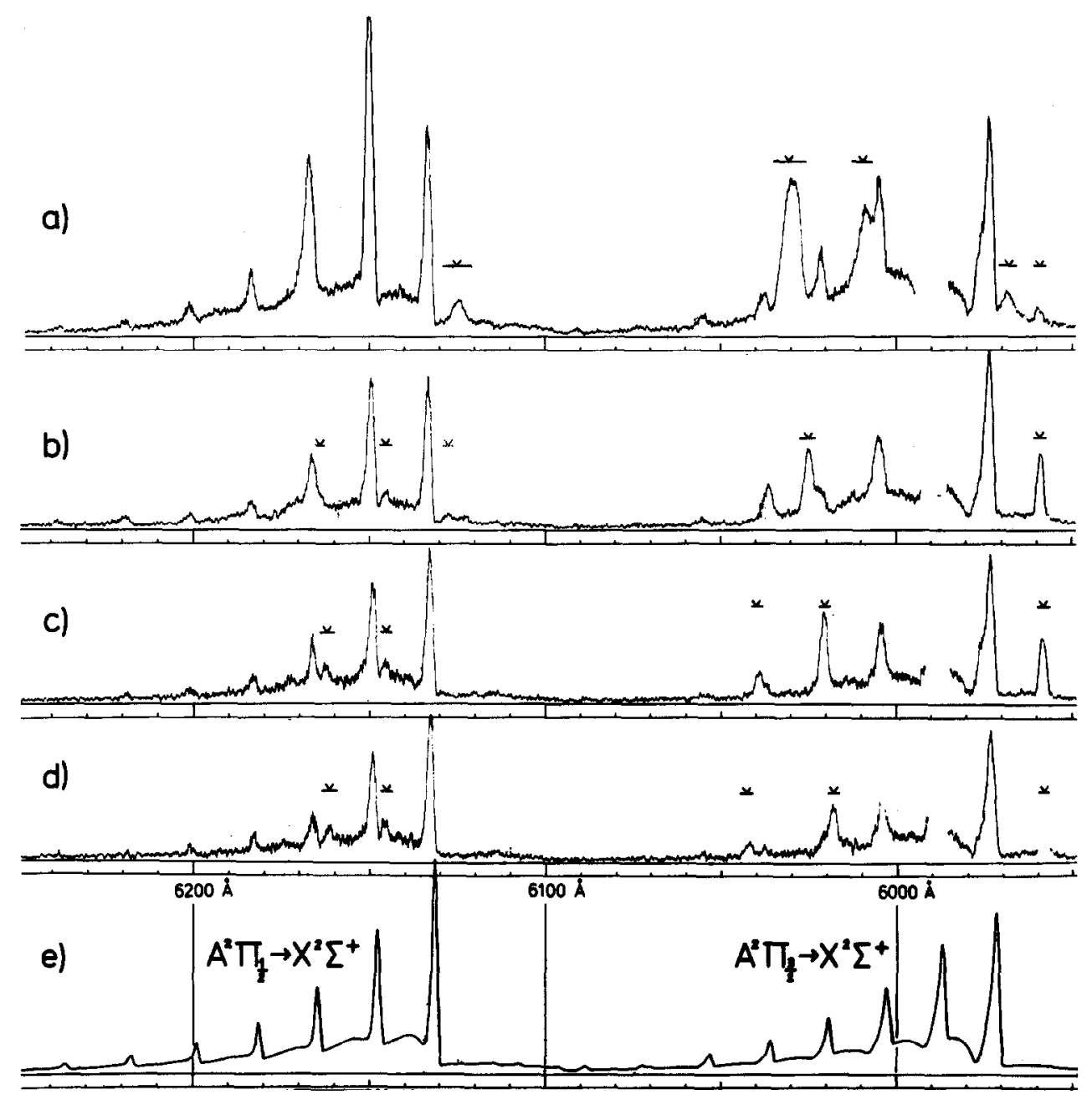

Fig. 5. Fluorescence emission spectra from single-line excitation. Spectra a, b, c and d resulted from the excitation of rotational lines in Fig. 4 marked with (a, b, c and d) respectively. In Fig. $5(e)$ the thermal emission spectrum is presented on the same wavelength scale.

not in the thermal emission spectrum. Shifting the wavelength of excitation to an adjacent line causes a shift of these structures on the blue side in the same $\lambda$-direction, whereas structures on the red side shift in the opposite direction.

4. The ratios between the spectrally integrated intensities of Q-branches belonging to different low $v$ numbers for each of the doublet components depend on which rotational line has been excited. Since the fluorescence spectrum clearly depends on the line selected for excitation, this single-line excitation is unsuitable for the study of vibrational relaxation effects only.

\subsection{Fluorescence emission spectra from multi-line excitation}

By sweeping through a strong Q-branch of a $v^{\prime} \rightarrow v^{\prime \prime}=v^{\prime}$ transition with the laser (see Section 2.4.4) at a repetition of about $0.5 \mathrm{~s}^{-1}$, we detected fluorescence visually with the equipment described in Section 2.3. When the laser line started its sweep in the blue $\lambda$-direction from the origin of the selected Q-branch, a faint fluorescence "band" also started from the origin but moved in the red $\lambda$-direction in agreement with Section 3.2 point 3 . On the blue side such a "band" was visible too, except when the $(0,0)$ Q-branch was excited. Resonance radiation was seen as well, particularly at the heads of strongest $(0,0) \mathrm{Q}$-branches.

Next we increased the sweep-repetition rate of the laser wavelength to $5 \mathrm{~Hz}$ in order to record the fluorescence spectra and the thermal emission spectra simultaneously as 
described in Section 2.4.4. The wavelength range of the laser was chosen about $10 \%$ larger than the wavelength distance from origin to the head of the selected Q-branch to ensure adequate coverage.

To ensure that the reproduction of steep slopes in both spectra was identical, the time constants of lock-in 1 as well as lock-in 2 were set the same, at $10 \mathrm{~s}$. The recording speed was $2 \AA \min ^{-1}$. A series of simultaneously recorded Q-branch spectra of the $\left(v^{\prime}=0, \ldots, 8, v^{\prime \prime}=v^{\prime}\right) A^{2} \Pi_{1 / 2} \rightarrow X^{2} \Sigma^{+}$and $\left(v^{\prime}=0, \ldots, 7, v^{\prime \prime}=v^{\prime}\right) A^{2} \Pi_{3 / 2} \rightarrow X^{2} \Sigma^{+}$transitions were obtained by exciting one Q-branch.

Each of the above mentioned Q-branches was excited separately which resulted in 17 series of these simultaneously recorded spectra. As an illustration, Q-branch spectra of the $\left(v^{\prime}=0, \ldots, 5, v^{\prime \prime}=v^{\prime}\right) \quad A^{2} \Pi_{3 / 2} \rightarrow X^{2} \Sigma^{+}$transitions, where the Q-branch $(5,5)$ $A^{2} \Pi_{1 / 2} \rightarrow X^{2} \Sigma^{+}$was excited, are shown in Fig. 6. There fluorescence from Q-branches with $v$ numbers smaller than those of the laser-excited Q-branch shows only a weak dependence on $v$. Note the different amplification factors used in the recording of the two spectra and the $-0.07 \AA$ shift of the thermal emission spectrum recording, which is due to the different second-channel pen position.

In a fluorescence emission spectrum which encloses the full region between 5950 and $6280 \AA$ where the Q-branch of the $(1,1) A^{2} \Pi_{3 / 2} \rightarrow X^{2} \Sigma^{+}$transition was broad-band excited, the differences mentioned in points 1 and 2 of Section 3.2 appear too. Structures in fluorescence emission spectra found with single-line excitation appeared at about the same $\lambda$-position in fluorescence emission spectra with multi-line excitation; however structures in the latter spectra were broader. The appearance of these broader structures can be understood since strongest absorption takes place in a relatively small number of rotational lines at the head of the Q-branch.

So far no attention has been paid to the presence of P- and R-branches of different $v^{\prime} \rightarrow v^{\prime \prime}=v^{\prime}$ levels which partially overlap the excited Q-branch. Consequently, fluorescence from levels with different $v$ numbers due to collisional transfer as well as direct optical population may be expected. The fluorescence spectra need to be corrected for the latter contribution. At present we are trying to find a suitable theoretical or experimental way of carrying out this correction.

Note: From a thermal emission spectrum of $\mathrm{YO}$ we were able to determine FranckCondon factors of some $A^{2} \Pi_{3 / 2} \rightarrow X^{2} \Sigma^{+}$transitions assuming that the electronic transition moment and the rotational line distribution is the same in all the measured Q-branches. LIU and PARsons [8] obtained the following calculation results of YO: $q_{00}=0.99$ and $q_{55}=0.88$ as a mean for $J=0, \ldots, 75$. We found $0.995<q_{00}<1$ and $q_{55}=0.90 \pm 0.02$, which agrees well.

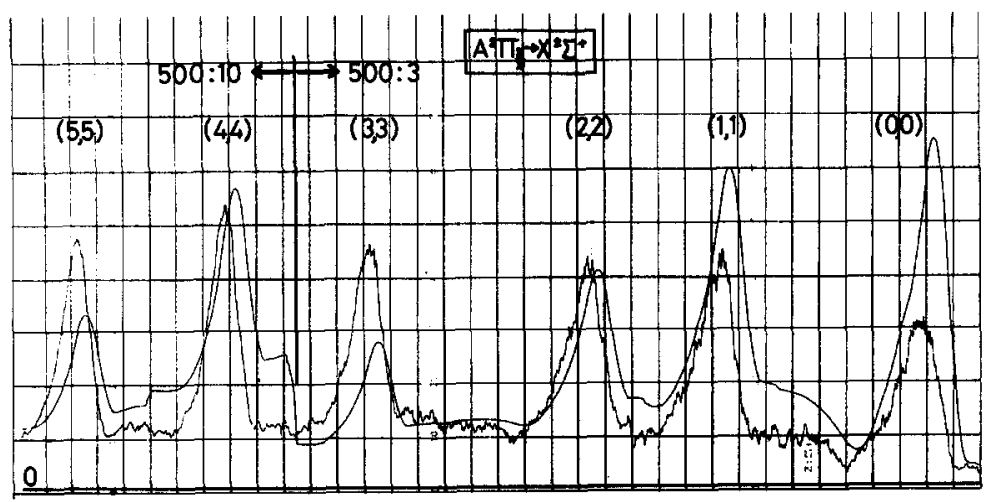

Fig. 6. Simultaneously recorded fluorescence spectrum (curve with clearly visible noise signal) and thermal emission spectrum plus fluorescence emission spectrum (smooth curve) of the Q-branches $\left(v^{\prime}=0, \ldots, 5, v^{\prime \prime}=v^{\prime}\right)$ of the $A^{2} \Pi_{3 / 2} \rightarrow X^{2} \Sigma^{+}$transition with broadband excitation of the Q-branch $(5,5)$ of the $A^{2} \Pi_{1 / 2} \rightarrow X^{2} \Sigma^{+}$transition. Note the amplification ratios between the two spectra.

[8] K. Lu, and J. M. Parson, J. Chem. Phys. 67, 1814 (1977). 


\section{Conclusions}

(a) The population distribution among the $v$-levels resulting from inelastic collisions following laser excitation as seen from the fluorescence spectrum is apparently not in partial thermal equilibrium. This deviation from a partial Boltzmann population mentioned in point 1 in Section 3.2 enables us to determine relative transition rates between the $v$-levels. Moreover, this deviation indicates that the collisional rate of relaxation to thermal equilibrium among the $v$-levels is smaller than the de-excitation rate.

(b) From point 2 in Section 3.2 we can conclude that redistribution among the doublet levels has been almost completed before de-excitation occurs. Hence the doublet mixing rate is larger than the de-excitation rate. If the quenching rate is not much smaller than this de-excitation rate, then conclusion (b) is qualitatively in agreement with results found for $\mathrm{BaCl}$ [9].

(c) The energy transferred in the doublet mixing process is large compared to the energy difference between the rotational levels. Hence values of $\Delta J$ in the simultaneously occurring rotational transitions will reach from zero to a number that is large enough for an efficient redistribution among the rotational levels in the non-excited doublet level. This is in agreement with point 3 in Section 3.2.

(d) From point 1 and the result in Fig. 6 we can conclude that a collisional transition from a vibrational level downward is more likely than a collisional transition with the same difference in energy to a higher vibrational level. This is qualitatively in agreement with the principle of detailed balance applicable to this flame.

(e) Regarding the great number of rotational lines in the Q-branches where excitation successfully takes place with multi-line excitation (Section 3.3), we conclude that this type of excitation is suitable to determine ratio's between the thermal population of a vibronic level and the population increase of the same level due to laser excitation.

[9] S. A. Edelstein, D. L. Huestis and J. Wyss, J. Chem. Phys. 70, 131 (1979). 